\title{
The Forest Hiding Problem
}

\author{
Adrian Dumitrescu • Minghui Jiang
}

Received: 7 October 2009 / Revised: 9 April 2010 / Accepted: 15 April 2010 /

Published online: 8 May 2010

(C) Springer Science+Business Media, LLC 2010

\begin{abstract}
Let $\Omega$ be a disk of radius $R$ in the plane. A set $F$ of unit disks contained in $\Omega$ forms a maximal packing if the unit disks are pairwise interior-disjoint and the set is maximal, i.e., it is not possible to add another disk to $F$ while maintaining the packing property. A point $p$ is hidden within the "forest" defined by $F$ if any ray with apex $p$ intersects some disk of $F$, that is, a person standing at $p$ can hide without being seen from outside the forest. We show that if the radius $R$ of $\Omega$ is large enough, one can find a hidden point for any maximal packing of unit disks in $\Omega$. This proves a conjecture of Joseph Mitchell. We also present an $O\left(n^{5 / 2} \log n\right)$-time algorithm that, given a forest with $n$ (not necessarily congruent) disks, computes the boundary illumination map of all disks in the forest.
\end{abstract}

Keywords Illumination problem $\cdot$ Maximal disk packing $\cdot$ Blocking light rays

\section{Introduction}

In this article we solve an illumination problem for maximal unit disk packings. A unit disk is a disk of unit diameter. Let $\Omega$ be a disk of radius $R$ in the plane.

A preliminary version of this paper appeared in the Proceedings of the Twenty-First Annual ACM-SIAM Symposium on Discrete Algorithms (SODA 2010), pp. 1566-1579.

The research of A. Dumitrescu was supported in part by NSF CAREER grant CCF-0444188. The research of M. Jiang was supported in part by NSF grant DBI-0743670.

\footnotetext{
A. Dumitrescu $(\varangle)$

Department of Computer Science, University of Wisconsin-Milwaukee, Milwaukee, WI 53201-0784, USA

e-mail: ad@cs.uwm.edu

M. Jiang

Department of Computer Science, Utah State University, Logan, UT 84322-4205, USA

e-mail: mjiang@cc.usu.edu
} 
Fig. 1 A dense forest with congruent trees. Two dark points (drawn as filled circles) and two illuminated points (drawn as empty circles) on some disk boundaries are shown. The four common tangents to a pair of disks are drawn with solid lines. Tangent segments (drawn with dotted lines) that intersect other disks are discarded by the algorithm for computing the boundary illumination map

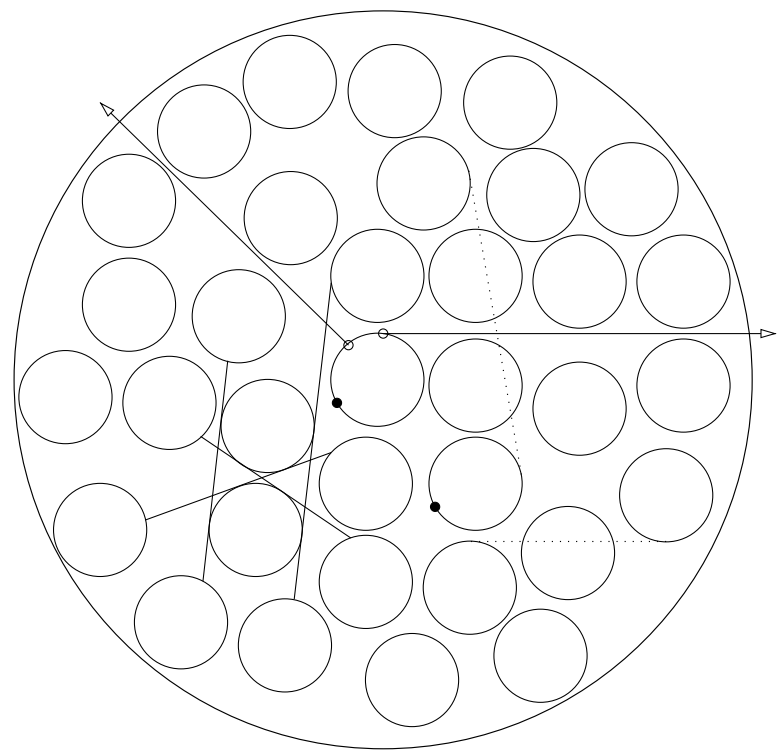

A set $F$ of unit disks in the plane contained in $\Omega$ forms a maximal packing if the unit disks are pairwise interior-disjoint and the set is maximal, i.e., it is not possible to add another disk to $F$ while maintaining the packing property. A finite packing of (arbitrary radii) disks is referred to as a forest. A maximal packing of unit disks in $\Omega$ is referred to as a dense forest. See Fig. 1. A point $p$ is dark within the forest defined by $F$ if any ray with apex $p$ intersects some disk of $F$, i.e., a person standing at $p$ cannot see out of the forest, or equivalently, he/she cannot be seen from outside the forest. A dark point is also referred to as a hidden point. A point that is not dark is illuminated. Consider any dense forest (with congruent disks) contained in $\Omega$. We show that if the radius $R$ of the forest is large enough, then there exists a dark point in $\Omega$ on some unit disk boundary. This answers an inspiring open problem posed by Joseph Mitchell at the 2007 Fall Workshop in Computational Geometry [10].

Theorem 1 Any dense (circular) forest with congruent trees (of unit diameter) that is deep enough has a hidden point. More precisely, if the forest radius $R$ is large enough, namely $R \geq 2 \cdot 10^{108}$, then there exist hidden points on the boundaries of at least $R^{2} / 14$ disks.

Let $R^{*}$ be the supremum of such $R$ for which a hidden point is not guaranteed to exist, i.e., there exists a forest of radius $R$ such that all points on the boundaries or in between the disks are illuminated. Our Theorem 1 yields $R^{*} \leq 2 \cdot 10^{108}$, and we preferred simplicity to sharpness in our arguments. On the other hand, we would be surprised if $R^{*}>100$ were to hold.

It is interesting to note that with square trees, one can plant a forest that does not have a hidden point, no matter how deep and how dense it is; see Fig. 2. It is a good exercise for the reader to determine which part in the proof of Theorem 1 breaks down for this type of forest. 
Fig. 2 A deep and dense forest of square trees with no hidden point can be planted using this pattern

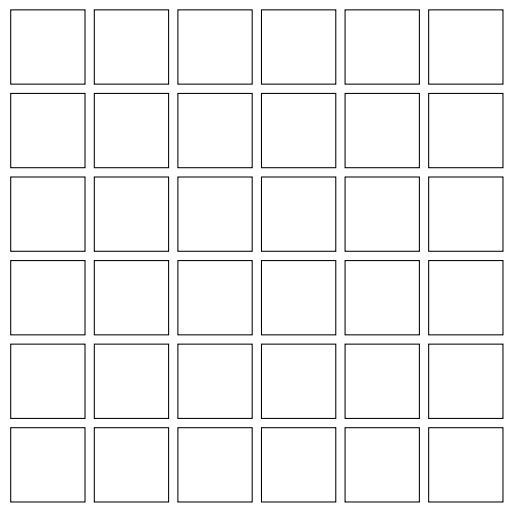

In the final part of our paper we outline an algorithm that, given a forest with $n$ (arbitrary) disks, determines for each disk boundary point, whether it is dark or illuminated. The illumination map of a forest $F=\left\{\omega_{1}, \ldots, \omega_{n}\right\}$ in $\Omega$ is a binary function which specifies for each point in the free space among the trees, $\Omega \backslash \bigcup_{i=1}^{n} \operatorname{int}\left(\omega_{i}\right)$, whether it is dark or illuminated. The boundary illumination map of a forest is the restriction of the above function to the boundaries of the disks in $F$, that is, it specifies for each point $p \in \partial \omega$, where $\omega \in F$, whether it is dark or illuminated.

Theorem 2 Given a (not necessarily dense) forest with $n$ (not necessarily congruent) disks, there exists an $O\left(n^{5 / 2} \log n\right)$-time algorithm that computes the boundary illumination map of (all disks in) the forest.

Related problems and results Perhaps the closest related problem to the forest hiding problem we study here is the classical orchard visibility problem due to Polya [13]. In this problem, a tree is planted at each lattice point except the origin, in a circular orchard which has center at the origin and radius $R$. The question is how thick the trees must be in order to block completely the visibility from the origin. If the radius of the trees exceeds $1 / R$ units, one is unable to see out of the orchard in any direction from the origin. However, if the radii of the trees are smaller than $1 / \sqrt{R^{2}+1}$, one can see out at certain angles. Various solutions due to Polya himself [13], Polya and Szego [14], Honsberger [5], Yaglom and Yaglom [15] have appeared over the years. Some new variants have been explored in $[2,6,7,9]$.

A problem "dual" to Mitchell's forest hiding problem, where the trees are reflecting light rather than blocking it, is Pach's enchanted forest problem with circular mirrors [3, p. 19], [11, p. 653]: If a match is lit in an enchanted forest of mirror trees, must some light escape to infinity? It is conjectured [12] that no finite collection of disjoint circular mirrors (i.e., a forest of mirror trees) can trap all the rays from a point light source. While Pach's question can be asked for any forest, the answer is not known even for forests with congruent trees (i.e., unit disks). 


\section{Preliminaries}

Let $F$ be a dense forest of unit disks contained in $\Omega$. If a point on a unit disk boundary is illuminated by multiple light rays, we pick one such ray arbitrarily. In our proof, we will find hidden points on the boundaries of disks in $F$.

Parameters We will use a set of carefully chosen and highly interdependent parameters, which we collect here for easy reference (recall that $R$ is the forest radius):

$$
\begin{aligned}
\phi & =\pi / 600, \quad \beta=\phi / 2=\pi / 1200, \quad \theta=\pi / 60 ; \\
\lambda & =1 / 146000, \quad \lambda_{1}=1 / 200000, \quad \delta=\kappa=\lambda_{1} / 3=1 / 600000 ; \\
R_{2} & =0.99 R, \quad R_{1}=R_{2} / \sqrt{2}, \quad L=2 R_{1} .
\end{aligned}
$$

Notation and definitions Let $\bar{r}$ be a light ray. Given two points $s, t \in \bar{r}$, where $s$ is closer to the light source, for convenience, we sometimes consider $\bar{r}$ anchored at $s$, and sometimes at $t$. Fix an arbitrary coordinate system. The direction vector of a light ray is the unit vector along the ray from the light source. In the plane, we identify each direction vector with its angle $\in[0,2 \pi)$. Further we subdivide the set of all directions in 1200 direction intervals of angle $\phi=2 \beta=0.3^{\circ}$ each: set $\beta=\pi / 1200$ and $I(\alpha)=[\alpha-\beta, \alpha+\beta), \alpha=i \pi / 600, i=0,1, \ldots, 1199$. Thus each direction interval $I(\alpha)=[\alpha-\beta, \alpha+\beta)$ is symmetric about its main direction $\alpha$. There are 1200 main directions of illumination and corresponding direction intervals symmetric about each of these main directions. Many of the arguments will be proven for $\alpha=3 \pi / 2$, for convenience, and without any loss of generality. A ray $\bar{r} \in I(3 \pi / 2)$ is said to be almost vertical.

Recall that $\Omega$ is a disk of radius $R$. Let $\Omega_{1}$ and $\Omega_{2}$ be concentric disks of radii $R_{1}$ and $R_{2}$, where $R_{2}=0.99 R$ and $R_{1}=R_{2} / \sqrt{2}$. Thus $R_{1}<R_{2}<R$. Let $L=2 R_{1}$. For each of the main directions $\alpha=i \pi / 600, i=0,1, \ldots, 1199$, let $Q_{\alpha}$ be a square of side $L$ and orientation $\alpha$ concentric with $\Omega$, i.e., an axis-aligned square rotated counterclockwise by $\alpha$. Refer to Fig. 3 for a generic example $(\alpha=0)$. By our setting of the parameters, we have

$$
L=2 R_{1}=\sqrt{2} R_{2}=0.99 \cdot \sqrt{2} R .
$$

For any of the main directions $\alpha$, observe the inclusions:

$$
\Omega_{1} \subset Q_{\alpha} \subset \Omega_{2} \subset \Omega
$$

Let $\gamma<1 / 2$. Given a segment $s$, the $\gamma$-central part of $s$ is the smaller segment of length $(1-2 \gamma)|s|$ obtained from $s$ by cutting off a small segment of length $\gamma|s|$ from each end.

A disk in $F$ is said to be totally illuminated if all its boundary points are illuminated by light rays. Otherwise the disk is said to be partially illuminated.

The following distinction among light rays illuminating disks is crucial in our arguments. Let $\bar{r}$ be a light ray that illuminates a point on the boundary of a unit disk $\omega$. We say that $\bar{r}$ frontally illuminates $\omega$ if the extension of $\bar{r}$ in the interior of the 
Fig. 3 A generic example with $\alpha=0$
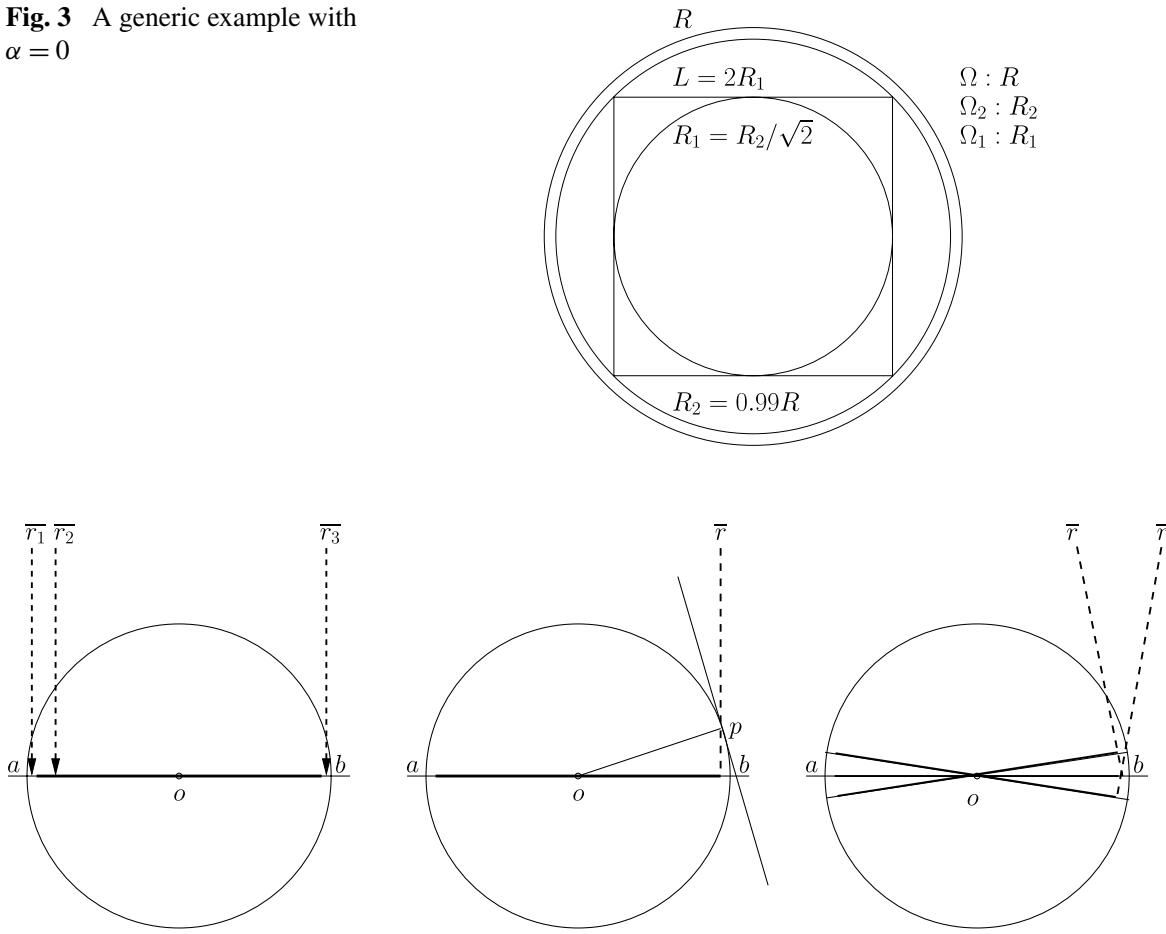

Fig. 4 Left: two tangential vertical rays $\left(\overline{r_{1}}\right.$ and $\left.\overline{r_{3}}\right)$ and a frontal vertical ray $\overline{r_{2}}$ illuminating a unit disk from above. The central part of the horizontal diameter $a b$ is drawn with a thicker line. Middle: a tangential ray must make an angle at most $\phi$ with the tangent at the contact point $p$. Right: two rays in the interval $I(\alpha)$, where $\alpha=3 \pi / 2$, which frontally illuminate the disk from above

disk meets the $\lambda$-central part of the diameter of $\omega$ orthogonal to $\bar{r}$; otherwise we say that $\bar{r}$ tangentially illuminates $\omega$. Correspondingly, we refer to the two types of rays as frontal rays and, respectively, tangential rays. See Fig. 4 (left). A totally illuminated unit disk $\omega$ is said to be frontally illuminated if at least one point on its boundary is frontally illuminated. A totally illuminated unit disk $\omega$ is said to be tangentially illuminated if all points on its boundary are tangentially illuminated. Two important properties of these types of disk illumination are proved in Lemma 1.

Lemma 1 Let $\omega$ be a unit disk.

(i) Let $\bar{r}$ be a ray that tangentially illuminates $\omega$. Then the angle between $\bar{r}$ and the tangent to $\omega$ at the contact point $p$ is at most $\phi$.

(ii) Let $\bar{r}$ be a ray that frontally illuminates $\omega$, with $\bar{r}$ in the interval $I(\alpha)$, for some main direction $\alpha$. Then the extension of $\bar{r}$ in the interior of the disk meets the $\lambda_{1}$-central part of the diameter of $\omega$ orthogonal to direction $\alpha$.

Proof Let $a b$ be the horizontal diameter segment, and let $o$ be the center of $\omega$.

(i) See Fig. 4 (middle). Assume for convenience that the ray is vertical. By definition, $\bar{r}$ meets $a b$ at a point at distance at most $\lambda$ from point $b$. Connect $o$ with $p$ and 
observe that the angle between $\bar{r}$ and the tangent at $p$ is the same as the angle $\angle p o b$. We have

$$
\begin{aligned}
\angle p o b & \leq \arccos \left(\frac{\frac{1}{2}-\lambda}{\frac{1}{2}}\right)=\arccos (1-2 \lambda)=\arccos \left(1-\frac{1}{73000}\right) \\
& =0.29990 \ldots{ }^{\circ}<0.3^{\circ}=\phi .
\end{aligned}
$$

(ii) See Fig. 4 (right). Assume for convenience that the main direction $\alpha$ is vertical, i.e., $\alpha=3 \pi / 2$. By definition of direction intervals, the angle between $\bar{r}$ and direction $\alpha$ is at most $\beta$. It follows that the horizontal distance from the intersection of (the extension of) $\bar{r}$ and $a b$ to point $b$ is at least

$$
\frac{1}{2}-\frac{\frac{1}{2}-\lambda}{\cos \beta}=\frac{1}{2}-\frac{\frac{1}{2}-\frac{1}{146000}}{\cos \frac{\pi}{1200}}=0.000005135 \ldots>\frac{1}{200000}=\lambda_{1} .
$$

We now establish two basic properties that follow from the maximality of the disk packing (Lemmas 2 and 3).

Lemma 2 Let $U$ be a square of side 2 contained in $\Omega$. Then $U$ contains the center of at least one disk in $F$ in its interior.

Proof Denote by $\xi$ the center of $U$. Assume for contradiction that the center of each disk in $F$ intersecting $U$ lies outside $U$ or on $\partial U$. Then the open unit square $V$ with center $\xi$ and homothetic to $U$ does not intersect any disk in $F$. Consequently, the unit disk $\omega(\xi)$ centered at $\xi$ is contained in $V$ and is interior-disjoint from any unit disk in $F$, which in turn contradicts the maximality of the packing.

Lemma 3 Assume that $R \geq 1000$. Then there are at least $3 L^{2} / 16$ disks from $F$ in $\Omega_{1}$.

Proof The area of $\Omega_{1}$ is $\pi R_{1}^{2}$. Superimpose an axis-aligned grid of squares of side 2 over $\Omega_{1}$ with one of the squares centered at the center $o$ of $\Omega_{1}$. The squares partition the area inside $\Omega_{1}$. Observe that the number of squares of side 2 that are contained in $\Omega_{1}$ and are at distance at least $1 / 2$ from its boundary equals the number of even lattice points (i.e., those with both coordinates even) contained in a circle of radius $R_{1}-$ $\sqrt{2}-1 / 2$ centered at $o$. This number equals the number of lattice points contained in a circle of radius $r=\left(R_{1}-\sqrt{2}-1 / 2\right) / 2$ centered at $o$. By a well-known result of Gauss [4, p. 67], this number is at least $\pi r^{2}-2 \sqrt{2} \pi r$. A straightforward calculation shows that, for $R \geq 1000$, we have

$$
\pi r^{2}-2 \sqrt{2} \pi r \geq 3.1 r^{2} \geq \frac{3 R_{1}^{2}}{4}=\frac{3 L^{2}}{16}
$$

By Lemma 2, every one of these squares contains the center of at least one unit disk in $F$, and thus there are at least $3 L^{2} / 16$ disks from $F$ in $\Omega_{1}$. 


\section{Proof of Theorem 1}

Proof outline In a nutshell, the proof of Theorem 1 goes as follows. In Lemma 8 we prove that no disk in $\Omega_{1}$ is tangentially illuminated. Assuming tangential illumination for such a disk would imply the existence of a "hole" in the packing, a contradiction to its maximality. To make this verification easy, we have included a short computer program for computing intersection points between a suitable set of seven rays almost tangent to a disk and three parallel lines. We defer the rather long proof of this technical lemma to the end of Sect. 3.

Second, we prove in Lemma 7 that the number of frontally illuminated disks in a relatively large disk $\Omega_{1}$ (of radius $R_{1}$ ) concentric with $\Omega$ is at most a constant fraction (less than 1) of all the disks in $\Omega_{1}$. This part is the most difficult in the proof, relying on the sequence of Lemmata 4 through 7 . It then follows that the remaining disks in $\Omega_{1}$ (a constant fraction of all disks) are only partially illuminated, and therefore each has a hidden point on its boundary. We conclude that for large enough $R$, there exist many (that is, $c R^{2}$ for an absolute constant $c>0$ ) distinct hidden points, each associated with a different disk in $F$. We now proceed with the details. Section 3.1, on frontal illumination is devoted to Lemmata 4 through 7. Section 3.2, on tangential illumination is devoted to Lemma 8.

\subsection{Frontal Illumination}

We need the following weight distribution lemma. Some intuition is provided below. Given a (rectangular) region $V$ and a subset $F^{\prime} \subseteq F$ of the disk packing, the weight of $V$ is the number of disk centers from $F^{\prime}$ that lie in $V$. Recall the setting of $L$ in (1). Let $Q$ be an axis-aligned square of side length $L$ with weight $W=\Theta\left(L^{2}\right)$. An axis-aligned subrectangle $V \subset Q$ of width $L$ and height $h$ is called a separated heavy strip in $Q$ if: (i) the rectangle in $Q \backslash V$ of width $L$ bordering $V$ from above has height $h$, and (ii) the weight of $V$ is $\Theta\left(L^{2}\right)$.

Lemma 4 shows the existence of a separated heavy strip under a suitable assumption. The reason we want a separated heavy strip $H$ is the following: Assuming that many disks, $c L^{2}$, from $H$ are frontally illuminated from above (almost vertically), by the pigeonhole principle, we can find $c^{\prime} L$ rays that are anchored on the top side of $Q$ in some short common interval $K$ (for practical purposes, $K$ acts as a single point). Now, a separated strip enforces a separated point set on the top line of $H$ (Lemma 5). In this context, a separated point set on the line has the minimum pairwise distance bounded from below by a positive constant.

Lemma 4 Assume that $R \geq 2^{16}$. Let $F^{\prime} \subseteq F$ be a subset of at least $W=L^{2} / 8000$ disks. Let $Q$ be an axis-aligned square of side length $L$ containing all the disks in $F^{\prime}$. Then there exists a separated heavy horizontal strip $H \subset Q$ of height at least $L / 2^{15}$ that contains at least $W / 30=L^{2} / 240000$ disks in $F^{\prime}$.

Proof Refer to Fig. 5 for a smaller example. We have $W=L^{2} / 8000$. Since $L \geq R$ (recall (1)), we also have $L \geq 2^{16}$. Consider a partition of $Q$ into 16 horizontal strips of width $L$. The strips are labeled $H_{0}$ through $H_{15}$ in top to bottom order. The height of $H_{0}$ is $L / 2^{15}$. For $i=1, \ldots, 15$, the height of $H_{i}$ is $L / 2^{16-i}$. 
Fig. 5 Partition of $Q$ into 5 horizontal strips. Each of the strips $\mathrm{H}_{1}, \mathrm{H}_{2}, \mathrm{H}_{3}, \mathrm{H}_{4}$ can be a separated heavy strip if its weight is $\Theta\left(L^{2}\right)$

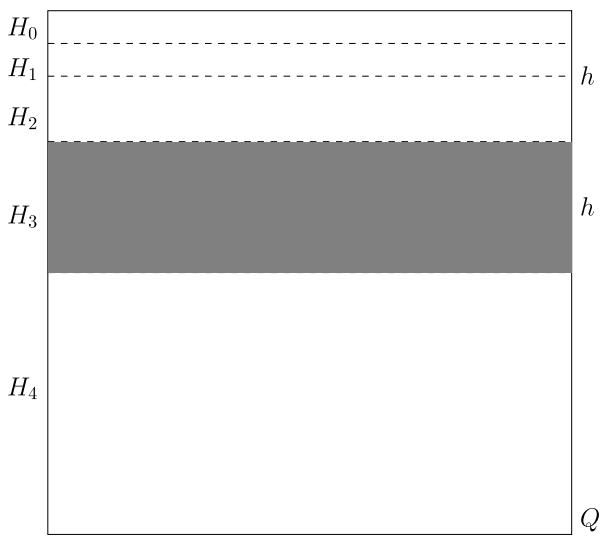

We distinguish two cases: If any of the strips $H_{1}$ through $H_{15}$ has weight at least $W / 30$, we have found a separated heavy strip, and we are done: (i) the strip has at least $L^{2} / 240000$ disk centers, and (ii) its height is $L / 2^{16-i} \geq L / 2^{15}$. So assume then that each of the 15 strips has weight smaller than $W / 30$. Their total weight is less than $15 W / 30=W / 2$. It follows that the weight of $H_{0}$ is at least $W-W / 2=W / 2=$ $L^{2} / 16000$. All disks whose centers lie in $H_{0}$ are contained in rectangle $H_{0}^{\prime} \supset H_{0}$ that extends $H_{0}$ up and down by $1 / 2$ (recall that disks could be "cut" only by horizontal strip boundaries). Thus $H_{0}^{\prime}$ contains at least $L^{2} / 16000$ disks. On the other hand, if $L \geq 2^{16}$, the height of $H_{0}^{\prime}$ is only

$$
\frac{L}{2^{15}}+1 \leq \frac{3 L}{2^{16}}
$$

Since the disks form a packing, it follows by a simple area argument that the number of disks contained in $H_{0}^{\prime}$ is at most

$$
L\left(\frac{L}{2^{15}}+1\right) \cdot \frac{4}{\pi} \leq \frac{3 L^{2}}{2^{16}} \cdot \frac{4}{\pi} \leq \frac{L^{2}}{17000}<\frac{L^{2}}{16000} .
$$

We have reached a contradiction, so the latter case cannot occur.

A key step is the following lemma which shows a separation distance on $\ell_{2}$ of the ray intersection points for almost vertical rays anchored in a small interval $K$ on $\ell_{1}$.

Lemma 5 Let $\omega_{1}$ and $\omega_{2}$ be two unit disks whose centers lie in a separated heavy horizontal strip $H$ contained in an axis-aligned square $Q$. Let $\ell_{1}$ and $\ell_{2}$ be the horizontal lines through the top side of $Q$ and the top side of $H$, respectively. Consider two almost vertical rays, $\overline{r_{i}}, i=1,2$, such that $\overline{r_{i}}$ frontally illuminates $\omega_{i}$ from above, and $\overline{r_{i}}$ is anchored at point $s_{i} \in K$, where $K$ is a horizontal segment of length $|K|=\kappa$ on $\ell_{1}$. If $p_{i}$ denotes the intersection of the ray $\overline{r_{i}}$ with the line $\ell_{2}, i=1,2$, then $\left|p_{1} p_{2}\right| \geq \delta=1 / 600000$. 
Fig. 6 Illustration of Case 1 in the proof of Lemma 5

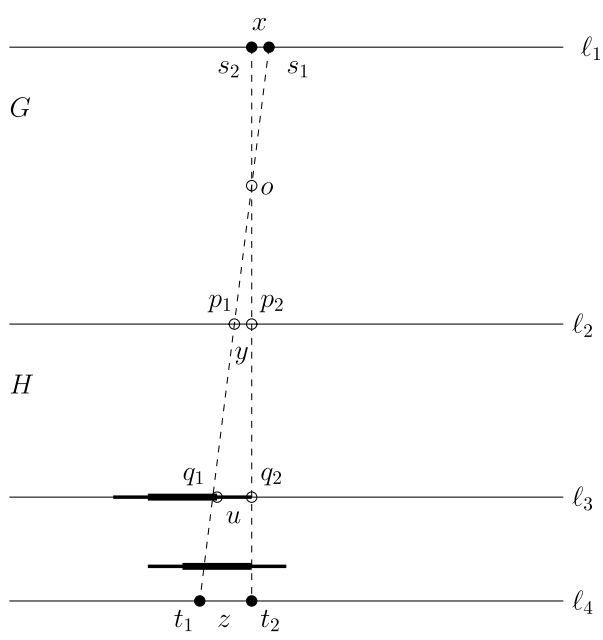

Proof Refer to Fig. 6. Denote by $h$ the height of $H$. Let $G$ be the horizontal strip of height $h$ that separates $H$ from the top side of $Q$. Assume without loss of generality that $\omega_{1}$ is at least as high as $\omega_{2}$ in $H$. Let $\ell_{3}$ and $\ell_{4}$ be the horizontal lines through the center of $\omega_{1}$ and the bottom side of $H$, respectively. Let $q_{i}$ be the intersection point of the ray $\overline{r_{i}}$ with $\ell_{3}$, and let $t_{i}$ be the intersection point of the extension of $\overline{r_{i}}$ with $\ell_{4}$. Write $x=\left|s_{1} s_{2}\right|, y=\left|p_{1} p_{2}\right|, u=\left|q_{1} q_{2}\right|$, and $z=\left|t_{1} t_{2}\right|$. By Lemma 1(ii), since both disks are frontally illuminated by almost vertical rays, we have $u \geq \lambda_{1}$. We distinguish two cases:

Case 1: $s_{1} t_{1}$ and $s_{2} t_{2}$ cross inside the rectangle $H \cup G$. Denote by $o$ the crossing point of $s_{1} t_{1}$ and $s_{2} t_{2}$, and let $h_{i}$ denote the vertical distance from $o$ to $\ell_{i}, i=1,2,3$. First observe that $o$ does not lie below $\ell_{3}$, since otherwise we would have, by the similarity of the triangles $\triangle o s_{1} s_{2}$ and $\triangle o q_{1} q_{2}$,

$$
\frac{x}{u}=\frac{h_{1}}{h_{3}}=\frac{h+h_{2}}{h_{3}} \geq \frac{2 h_{2}}{h_{3}} \geq \frac{2 h_{3}}{h_{3}}=2,
$$

and thus $x \geq 2 u \geq 2 \lambda_{1}$, contradicting the assumed inequality $x \leq \kappa=\lambda_{1} / 3$.

We can therefore assume that the crossing point $o$ lies above the horizontal line $\ell_{3}$. By the similarity of the triangles $\triangle o s_{1} s_{2}$ and $\triangle o q_{1} q_{2}$, we have

$$
\frac{h_{1}}{h_{3}}=\frac{x}{u} \leq \frac{\kappa}{\lambda_{1}}=\frac{1}{3} \text {. }
$$

Hence $h_{1} \leq h_{3} / 3 \leq 2 h / 3<h$, which means that $o$ lies above $\ell_{2}$ (i.e., in the strip $G$ ); see Fig. 6.

By the similarity of the triangles $\triangle o s_{1} s_{2}$ and $\triangle o t_{1} t_{2}$, and respectively of the triangles $\triangle o p_{1} p_{2}$ and $\triangle o t_{1} t_{2}$, we have

$$
\frac{h_{1}}{2 h-h_{1}}=\frac{x}{z} \quad \text { and } \quad \frac{y}{z}=\frac{h-h_{1}}{2 h-h_{1}} .
$$


From the first equality it follows that

$$
\frac{h_{1}}{h}=\frac{2 x}{x+z} .
$$

By plugging this value in the second equality, we get

$$
y=z \cdot \frac{h-h_{1}}{2 h-h_{1}}=z \cdot \frac{z-x}{2 z}=\frac{z-x}{2} \geq \frac{u-x}{2} \geq \frac{\lambda_{1}-\kappa}{2}=\kappa=\frac{1}{600000} .
$$

Case 2: $s_{1} t_{1}$ and $s_{2} t_{2}$ do not cross inside $H \cup G$. First observe that $s_{1} t_{1}$ and $s_{2} t_{2}$ cannot be parallel, since then $x=y=u \geq \lambda_{1}$, which contradicts $x \leq \kappa=\lambda_{1} / 3$. Similarly with previous arguments, $s_{1} t_{1}$ and $s_{2} t_{2}$ do not cross below $\ell_{4}$, since this would imply that $x>u \geq \lambda_{1}$, which contradicts $x \leq \lambda_{1} / 3$. If $s_{1} t_{1}$ and $s_{2} t_{2}$ cross above $\ell_{1}$, then it is easy to see that $y \geq u / 2 \geq \lambda_{1} / 2>\kappa=1 / 600000$, as required.

This completes the proof of Lemma 5 .

We have shown that frontal illumination with rays in the same direction interval of a large set of disks contained in a separated heavy strip leads to a dense set of separated points on a line. We first establish an approximate equidistribution lemma for such sets (Lemma 6). This in turn will lead to a small set of almost parallel rays going inside the forest and creating a "hole" in the packing, again in contradiction to its maximality (Lemma 7, to follow).

Lemma 6 Let $k$ be a positive integer, and $c, \delta>0$ be two positive constants. Let I be an interval of length $|I|$ containing at least $c|I|$ points $A=\left\{a_{1}, a_{2}, \ldots\right\}$ at least $\delta$ apart from each other. Put $r=\frac{k}{k-1}, j=\left\lceil\frac{\log \frac{2}{c \delta}}{\log r}\right\rceil$, and set $Z_{0}(k, c, \delta)=2 \delta \cdot k^{j}$. Then if $|I| \geq Z_{0}(k, c, \delta)$, there exists a sub-interval $J \subset I$ of length $k x$, for some $x \geq 2 \delta$, such that in the uniform subdivision of $J$ into $k$ equal half-closed sub-intervals $J_{1}, \ldots, J_{k}$ of length $x$, each sub-interval contains a distinct point in A.

Proof Conduct an iterative process as follows. In step 0: Subdivide the interval $I=I_{0}$ into $k$ half-closed intervals ${ }^{1}$ of equal length. If each of the $k$ intervals contains at least one point, stop. Otherwise at least one of the $k$ intervals is empty; now pick one of the remaining $k-1$ intervals, which contains the most points of $A$, say $I_{1}$. In step $i$, $i \geq 1$ : Subdivide $I_{i}$ into $k$ half-closed intervals of equal length and proceed as before.

In the current step $i$, the process either terminates successfully by finding an interval $I_{i}$ subdivided into $k$ sub-intervals, each containing at least one point in $A$, or it continues with another subdivision in step $i+1$. We show that if $|I|$ is large enough and the number of subdivision steps is large enough, the iterative process terminates successfully.

Let $L_{0}=|I|$ be the initial interval length, and $m_{0} \geq c|I|$ be the (initial) number of points in $I_{0}$. At step $i, i \geq 0$, let $m_{i}$ be the number of points in $I_{i}$, and let $L_{i}=\left|I_{i}\right|$ be

\footnotetext{
${ }^{1}$ When subdividing a closed interval, the first $k-1$ resulting subintervals are half-closed, and the $k$ th subinterval is closed. When subdividing a half-closed interval, all resulting subintervals are half-closed.
} 
the length of interval $I_{i}$. Clearly,

$$
L_{i}=\frac{|I|}{k^{i}}, \quad \text { and } \quad m_{i} \geq \frac{m_{0}}{(k-1)^{i}} \geq \frac{c|I|}{(k-1)^{i}} .
$$

Let $j$ be a positive integer such that

$$
c \cdot \delta \cdot\left(\frac{k}{k-1}\right)^{j} \geq 2, \quad \text { e.g., set } j=\left\lceil\frac{\log \frac{2}{c \delta}}{\log r}\right\rceil, \quad \text { where } r=\frac{k}{k-1} .
$$

Now set $Z_{0}(k, c, \delta)=2 \delta \cdot k^{j}$. If $|I| \geq Z_{0}$, as assumed, then by our choice of parameters we have

$$
L_{j}=\frac{|I|}{k^{j}} \geq \frac{2 \delta \cdot k^{j}}{k^{j}}=2 \delta
$$

and

$$
m_{j} \cdot \delta \geq \frac{c|I| \cdot \delta}{(k-1)^{j}}=c \cdot \delta \cdot\left(\frac{k}{k-1}\right)^{j} \cdot \frac{|I|}{k^{j}} \geq \frac{2|I|}{k^{j}}=2 L_{j} .
$$

Since the point set is $\delta$-separated, an interval packing argument on the line using (6) gives

$$
m_{j} \cdot \delta \leq L_{j}+\frac{\delta}{2}+\frac{\delta}{2}=L_{j}+\delta \leq \frac{3}{2} L_{j}
$$

Observe that (8) is in contradiction to (7), which means that the iterative process cannot reach step $j$. We conclude that for some $0 \leq i \leq j-1$, step $i$ is successful: we found an interval $J:=I_{i}$ of length $|J| \geq 2 k \delta$ such that each of its $k$ sub-intervals of length $|J| / k \geq 2 \delta$ contains at least one (distinct) point of $A$. This completes the proof.

The large upper bound on the forest radius in our result is mainly due to the following lemma, which in turn is a consequence of the large numbers $Z_{0}(k, c, \delta)$ in Lemma 6.

Lemma 7 Assume that $L \geq 380 \cdot Z_{0}\left(5,1 /\left(4.75 \cdot 10^{13}\right), 1 / 5\right)=760 \cdot 5^{151} \approx$ $2.66 \cdot 10^{108}$. For any direction interval $I(\alpha)$, at most $L^{2} / 8000$ disks in $Q_{\alpha}$ are frontally illuminated by rays in the interval $I(\alpha)$. Consequently, at most $3 L^{2} / 20$ disks in $\Omega_{1}$ are frontally illuminated.

Proof It is enough to prove the first statement for a given $\alpha$, say $\alpha=270^{\circ}=3 \pi / 2$. Write $Q=Q_{3 \pi / 2}$ and recall that we refer to rays in the interval $I(3 \pi / 2)$ as almost vertical rays. Let $\ell_{1}$ be the horizontal line through the top side of $Q$. Assume for contradiction that at least $W=L^{2} / 8000$ disks in $Q$ are frontally illuminated by almost vertical rays. By Lemma 4 with $F^{\prime}$ being this subset of disks, one can find a separated heavy strip $H$ of height $h$ containing at least $W / 30=L^{2} / 240000$ disks that are frontally illuminated by almost vertical rays (from above). Observe that all these rays are anchored on the top side of $Q$ extended by at most $L \tan \beta<L / 200$ in 
each direction, thus in an interval of length at most $1.01 \mathrm{~L}$. Recall that $\kappa=1 / 600000$ and divide the extended top side of $Q$ into at most $1.01 \mathrm{~L} / \kappa \leq 606000 \mathrm{~L}$ intervals of length $\kappa$. By the pigeonhole principle, at least

$$
\frac{L^{2}}{240000} \cdot \frac{1}{606000 L} \geq \frac{L}{1.5 \cdot 10^{11}}
$$

of these rays are anchored in some common interval $K=\left[k_{1} k_{2}\right] \subset \ell_{1}$ of length $|K|=\kappa$. Denote by $\mathcal{R}$ this set of rays. Consider the horizontal line $\ell_{2}$ through the top side of $H$. Observe that the rays in $\mathcal{R}$ intersects $\ell_{2}$ in an interval, say $I$ of length at most

$$
|I| \leq \kappa+2 \cdot \frac{L}{2} \cdot \tan \beta \leq \kappa+\frac{L}{381} \leq \frac{L}{380},
$$

since the vertical distance between $\ell_{1}$ and $\ell_{2}$ is at most $L / 2$. Denote by $A=a_{0}<$ $a_{1}<\cdots$ these intersection points on $\ell_{2}$. By Lemma 5, each pair of points $a_{i}<a_{j}$ are separated by at least $\delta=1 / 600000$ on $\ell_{2}$. Select points $a_{120000 i}, i=0,1, \ldots$, in total at least

$$
\frac{1}{120000} \cdot \frac{L}{1.5 \cdot 10^{11}}=\frac{L}{1.8 \cdot 10^{16}}
$$

points. The selected points have separation distance at least $\frac{120000}{600000}=\frac{1}{5}$ on $\ell_{2}$. For convenience, still denote by $A$ and respectively $\mathcal{R}$ this sparser subset of intersection points and corresponding rays. Observe that

$$
\frac{380}{1.8 \cdot 10^{16}} \geq \frac{1}{4.75 \cdot 10^{13}}, \quad \text { and set } k=5, c=\frac{1}{4.75 \cdot 10^{13}}, \delta=\frac{1}{5} .
$$

The calculation in Lemma 6 with these parameters yields:

$$
\begin{aligned}
& j=\left\lceil\frac{\log \left(4.75 \cdot 10^{14}\right)}{\log (5 / 4)}\right\rceil=152, \quad \text { and } \\
& 380 \cdot Z_{0}\left(5,1 /\left(4.75 \cdot 10^{13}\right), 1 / 5\right)=760 \cdot 5^{151} \approx 2.66 \cdot 10^{108} .
\end{aligned}
$$

By Lemma 6 , if $|I| \geq Z_{0}(k, c, \delta)$, or equivalently, $L \geq 380 \cdot Z_{0}(k, c, \delta)$, there exists an interval $J$ on $\ell_{2}$ of length $k x$, for some $x \geq 2 / 5$, such that in the uniform subdivision of $J$ into $k=5$ equal half-closed subintervals $J_{1}, \ldots, J_{5}$ of length $x$, each subinterval contains a distinct point in $A$. The $i$ th interval is $J_{i}=\left[j_{i}, j_{i+1}\right)$. Let $a_{1}<a_{2}<a_{3}<a_{4}<a_{5}$ be these five points on $\ell_{2}$, and let $s_{i} a_{i}, i=1,2,3,4,5$, denote these five rays in $\mathcal{R}\left(s_{i}, i=1,2,3,4,5\right.$, are the anchor points on $\ell_{1}$ of these rays). See Fig. 7 (left).

Let $\ell^{\prime}$ be a horizontal line (parallel to $\ell_{2}$ ) at vertical distance $2 h / 5 x$ from $\ell_{1}$. Let $j_{1}^{\prime}<j_{2}^{\prime}<j_{3}^{\prime}<j_{4}^{\prime}<j_{5}^{\prime}<j_{6}^{\prime}$ be the intersection points with $\ell^{\prime}$ of the six segments $k_{1} j_{i}$. Observe that these points form a uniform subdivision of the interval $\left[j_{1}^{\prime}, j_{6}^{\prime}\right]$ into five subintervals of length $2 / 5$. Observe that the segment $k_{1} a_{i}$ is a close approximation of the ray segment $s_{i} a_{i}$, where the latter can be obtained from the former by keeping the common endpoint $a_{i}$ fixed and moving the other endpoint on $\ell_{1}$ by at most $\kappa$. 


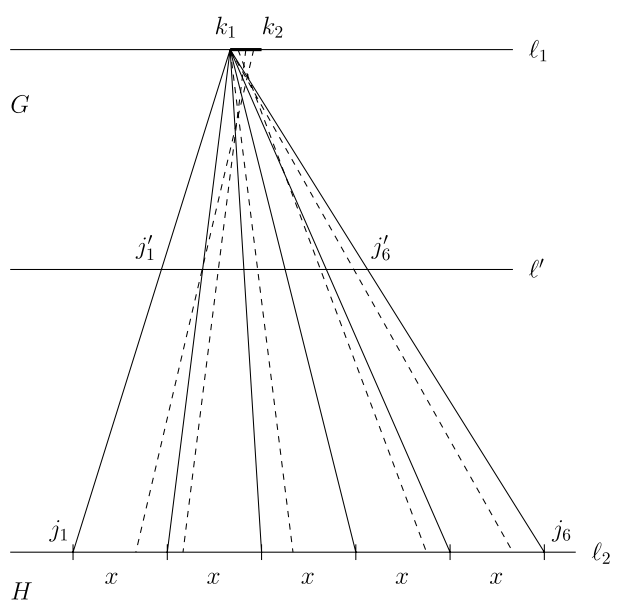

G

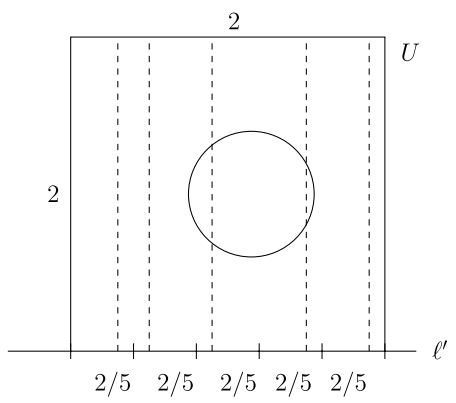

Fig. 7 Five rays almost uniformly distributed, and a square of side 2 contained in $\Omega$ and empty of unit disk centers. The light rays are drawn with dashed lines. The unit disk shown in the figure is infeasible

Consider now the axis-aligned square $U$ of side 2 based on $\ell^{\prime}$, with the two lower vertices at $j_{1}^{\prime}$ and $j_{6}^{\prime}$. See Fig. 7 (right). Note that $U$ is contained in $\Omega$. We claim that $U$ is empty of centers of disks in $F$. Let $p \in U$ be any point in this square. Observe that the distance from $p$ to one of the segments $k_{1} a_{i}$ is at most $2 / 5+2 \tan \beta<2 / 5+0.01=0.41$, and thus the distance from $p$ to one of the ray segments $s_{i} a_{i}$ is at most $0.41+\kappa<0.42<1 / 2$. This implies that a unit disk centered at $p$ intersects at least one of the five rays $s_{i} a_{i}$. Thus $p$ cannot be the center of a disk in $F$, and the emptiness claim for $U$ follows, in contradiction to Lemma 2. We conclude that the initial assumption that at least $L^{2} / 8000$ disks in $Q$ are frontally illuminated by almost vertical rays must be false. This proves the first statement of Lemma 7.

Recall (2) and (3). By summing over all 1200 direction intervals the above upper bound, we obtain that at most

$$
1200 \cdot \frac{L^{2}}{8000}=\frac{3 L^{2}}{20}
$$

disks in $\Omega_{1}$ are frontally illuminated. This completes the proof of Lemma 7.

\subsection{Tangential Illumination}

Note that if $R \geq 100$, then $R-R_{1}=R-0.99 R / \sqrt{2} \geq 20$.

Lemma 8 Assume that $R \geq 100$. Let $\omega \in F$ be a unit disk whose center is at distance at least 20 from the boundary of $\Omega$ (i.e., the center of $\omega$ lies in a disk of radius $R-20$ concentric with $\Omega$ ). Then $\omega$ is not tangentially illuminated.

Proof Assume for contradiction that $\omega$ is tangentially illuminated. Thus all its boundary points are tangentially illuminated. Consider a coordinate system in which the 
Fig. 8 The set

$P=\left\{p_{1}, p_{2}, p_{3}, p_{4}, p_{5}\right.$,

$\left.p_{6}, p_{7}\right\}$ on the boundary of $\omega$

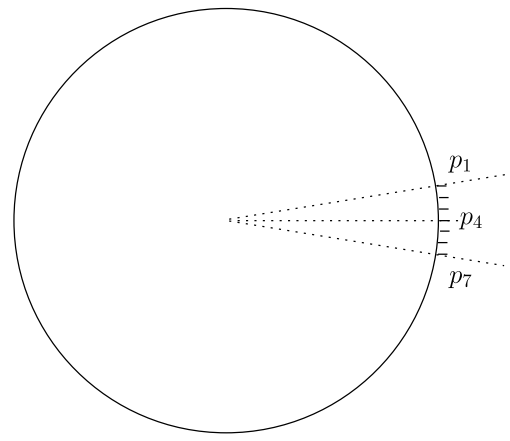

center of $\omega$ is $(-1 / 2,0)$. Let $\theta=\pi / 60$, and $\phi=\pi / 600$. Consider the following set of seven points on the boundary of $\omega, P=\left\{p_{1}, p_{2}, p_{3}, p_{4}, p_{5}, p_{6}, p_{7}\right\}$, where

$$
p_{i}=\frac{1}{2}(\cos (4-i) \theta-1, \sin (4-i) \theta), \quad i=1,2, \ldots, 7 .
$$

Observe that the seven points are equidistributed on a circular arc of $\omega$ subtending an angle of $6 \theta$, and symmetrically placed about the middle point $p_{4}=(0,0)$, as in Fig. 8.

Each of these points must be illuminated by an almost tangent ray pointing up, or by an almost tangent ray pointing down, where for convenience we see the rays anchored at the illuminated boundary points. Let $\overline{r_{i}}$ denote the ray illuminating $p_{i}$, $i=1,2, \ldots, 7$. By the pigeonhole principle, at least four rays are pointing in the "same" direction, and we can assume upwards, by symmetry. See also Fig. 9. Let $\mathcal{R}_{4}$ denote the set of these four rays. Consider a generic horizontal line $\ell=\ell(y)$, where $y \geq 3$. The ray $\overline{r_{i}}$ intersects $\ell(y)$ at point $\left(q_{i}, y\right), i=1,2, \ldots, 7$. For simplicity, we sometime omit the $y$-coordinate when it is not relevant and refer to the point simply as $q_{i}$. The points $\left(q_{i}, y\right), i=1,2, \ldots, 7$, on $\ell(y)$ are ordered from left to right (this will be apparent below).

Consider one of the upward rays, say $\overline{r_{i}}$. The fact that $\overline{r_{i}}$ is almost tangent to $\omega$ at $p_{i}$ implies that $q_{i} \in\left[a_{i}, b_{i}\right]$, where

1. $\left(a_{i}, y\right)$ is intersection point of $\ell(y)$ with the line tangent to $\omega$ at $p_{i}$, whose angle is $\pi / 2+(4-i) \theta$.

2. $\left(b_{i}, y\right)$ is the intersection point of $\ell(y)$ with a line incident to $p_{i}$ with angle $\pi / 2+$ $(4-i) \theta-\phi$, i.e., obtained by rotating the tangent line clockwise by $\phi$ around the (fixed) tangency point.

Observe that $a_{4}=0$. Note that $a_{i}=a_{i}(y), b_{i}=b_{i}(y)$, and $q_{i}=q_{i}(y)$ are linear functions of $y$. Further note that, for a fixed $i<j$, the differences $b_{j}-a_{i}$ and $a_{j}-b_{i}$ are increasing linearly with $y$. If $\overline{r_{i}}$ is pointing up, color $q_{i}$ red, otherwise color $q_{i}$ blue; the blue points correspond to missing upwards rays. Out of the seven points $q_{i}$ on $\ell$, at least four are colored red.

Proof plan. For each of the possibilities that can occur (in our case analysis below), we exhibit a square $U$ of side 2 contained in $\Omega$, that is empty of disk centers and thus is in contradiction with Lemma 2. 


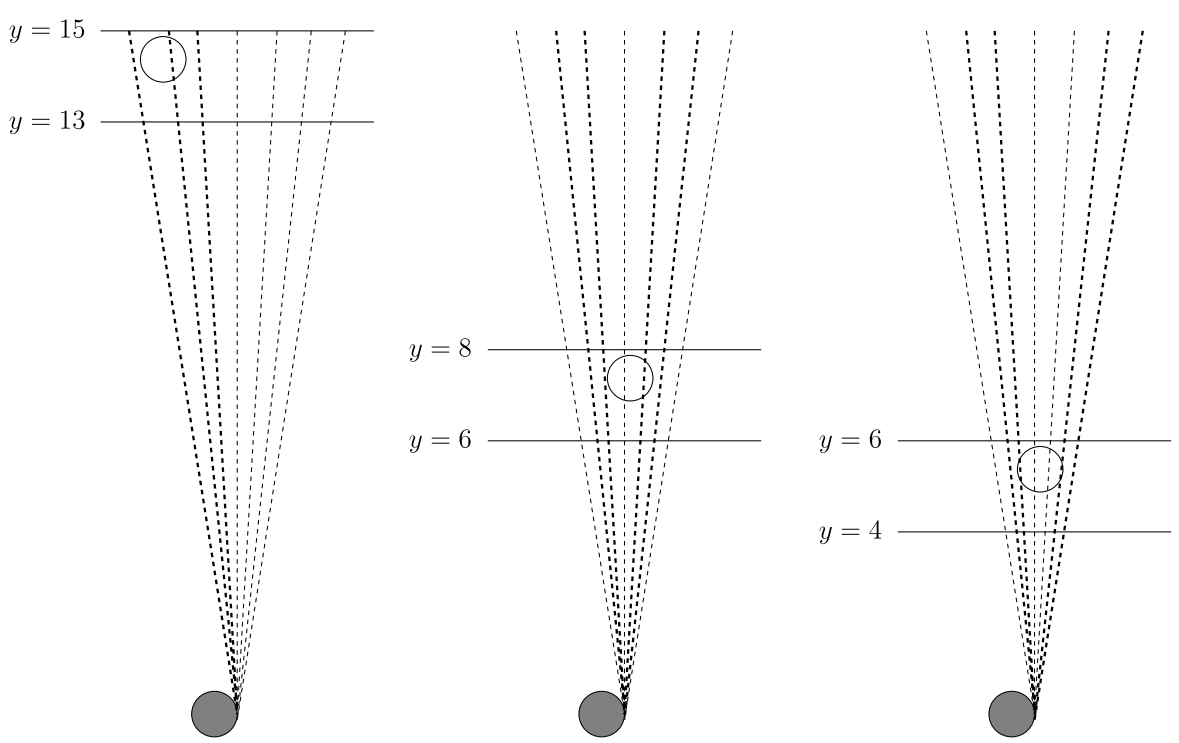

Fig. 9 Case 1.1 (on the left), Case 2 (in the middle), and Case 3 (on the right). The four rays in $\mathcal{R}_{4}$ are drawn with thicker dashed lines

Let the red span of $\ell$ be the maximum difference $j-i$, where $i<j$, and both $q_{i}$ and $q_{j}$ are red (this number is independent of $y$ ). Let the blue gap of $\ell$ be the maximum difference $j-i$, where $i<j$, both $q_{i}$ and $q_{j}$ are red, and all intermediate points (if any) $q_{i+1}, \ldots, q_{j-1}$ are blue (this number is also independent of $y$ ). We distinguish three cases:

Case 1: There exist three consecutive monochromatic points $q_{l}, q_{l+1}, q_{l+2}$ on $\ell$. Put $r=l+2$. By symmetry with respect to the $x$-axis, we can assume that they are red. Consider the two lines $\ell(15)$ and $\ell(13)$. A straightforward calculation ${ }^{2}$ gives the following values for $a_{i}(y)$ and $b_{i}(y), y=15, y=13$ :

$$
\begin{aligned}
& y=15: \quad a_{1}=-2.3695, \quad b_{1}=-2.2895 \text {, } \\
& a_{2}=-1.5738, \quad b_{2}=-1.4947 \text {, } \\
& a_{3}=-0.7854, \quad b_{3}=-0.7068, \\
& a_{4}=0.0000, \quad b_{4}=0.0785 \text {, } \\
& a_{5}=0.7868, \quad b_{5}=0.8657 \text {, } \\
& a_{6}=1.5793, \quad b_{6}=1.6590 \text {, } \\
& a_{7}=2.3820, \quad b_{7}=2.4630 \text {, } \\
& y=13: \quad a_{1}=-2.0528, \quad b_{1}=-1.9835 \text {, } \\
& a_{2}=-1.3636, \quad b_{2}=-1.2951 \text {, }
\end{aligned}
$$

\footnotetext{
${ }^{2}$ The calculations for all three cases can be reproduced by using the $\mathrm{C}$ program listed in the Appendix A.
} 


$$
\begin{array}{ll}
a_{3}=-0.6806, & b_{3}=-0.6125, \\
a_{4}=0.0000, & b_{4}=0.0681, \\
a_{5}=0.6820, & b_{5}=0.7504, \\
a_{6}=1.3691, & b_{6}=1.4382, \\
a_{7}=2.0652, & b_{7}=2.1355 .
\end{array}
$$

Observe that for $i=1,2,3,4,5,6$,

$$
b_{i+1}(15)-a_{i}(15) \leq b_{7}(15)-a_{6}(15)=0.8837<1 \text {. }
$$

Also, for $i=1,2,3,4,5$,

$$
a_{i+2}(13)-b_{i}(13) \geq a_{5}(13)-b_{3}(13)=1.2945>1 .
$$

We distinguish three subcases, depending on whether the slopes of the rays through $q_{l}$ and $q_{r}$ are (1) both negative, (2) both positive, or (3) one negative and one positive; see Fig. 9. We use the convention that a vertical ray has positive slope.

Case 1.1. $l=1$, and $r=3$ : the slopes of the rays through $q_{l}$ and $q_{r}$ are both negative. Consider the axis-aligned square $U_{1}$ of side 2 centered at $\xi_{1}=\left(\left(q_{l}(13)+\right.\right.$ $\left.\left.q_{r}(15)\right) / 2,14\right)$. We have

$$
q_{r}(15)-q_{l}(13) \geq a_{3}(15)-b_{1}(13)=1.1980>1 .
$$

By (9) and (10), the distance from any point $u \in U_{1}$ to one of the three rays $\overline{r_{l}}, \overline{r_{l+1}}$, or $\overline{r_{l+2}}$ is less than $1 / 2$. It follows that $U_{1}$ is empty of disk centers, in contradiction with Lemma 2.

Case 1.2. $l=2$, or $l=3$ : the slope of the ray through $q_{l}$ is negative, and the slope of the ray through $q_{r}$ is positive. Consider the axis-aligned square $U_{2}$ of side 2 centered at $\xi_{2}=\left(\left(q_{l}(13)+q_{r}(13)\right) / 2,14\right)$. We have

$$
q_{r}(13)-q_{l}(13) \geq a_{5}(13)-b_{3}(13)=1.2945>1 .
$$

It similarly follows that $U_{2}$ is empty of disk centers, in contradiction with Lemma 2.

Case 1.3. $l=4$ or $l=5$ : the slopes of the rays through $q_{l}$ and $q_{r}$ are both positive. Consider the axis-aligned square $U_{3}$ of side 2 centered at $\xi_{3}=\left(\left(q_{l}(15)+\right.\right.$ $\left.\left.q_{r}(13)\right) / 2,14\right)$. We have

$$
q_{r}(13)-q_{l}(15) \geq a_{7}(13)-b_{5}(15)=1.1995>1 .
$$

It follows in the same way that $U_{3}$ is empty of disk centers, in contradiction with Lemma 2.

For both Case 2 and Case 3, there are no three consecutive monochromatic points on $\ell$. In particular this means that the red span is at least 4 , and the blue gap is at most 3.

Case 2: There are no three consecutive monochromatic points on $\ell$, and the red span is 4 . Let $l<r$ be the two indices with $r-l=4\left(q_{l}\right.$ and $q_{r}$ are the leftmost and 
respectively the rightmost points on $\ell$ ). Observe that the ray through $q_{l}$ has negative slope and the ray through $q_{r}$ has positive slope. Consider the two lines $\ell(8)$ and $\ell(6)$. The corresponding values for $a_{i}(y)$ and $b_{i}(y), y=8, y=6$ are:

$$
\begin{aligned}
y=8: \quad a_{1}=-1.2608, & b_{1}=-1.2184, \\
a_{2} & =-0.8381, \quad b_{2}=-0.7960, \\
a_{3} & =-0.4186, \quad b_{3}=-0.3767, \\
a_{4} & =0.0000, \quad b_{4}=0.0419, \\
a_{5} & =0.4199, \quad b_{5}=0.4621, \\
a_{6} & =0.8436, \quad b_{6}=0.8862, \\
a_{7} & =1.2733, \quad b_{7}=1.3167, \\
y=6: \quad a_{1}=-0.9441, & b_{1}=-0.9123, \\
a_{2}=-0.6279, & b_{2}=-0.5964, \\
a_{3}=-0.3138, & b_{3}=-0.2824, \\
a_{4}=0.0000, & b_{4}=0.0314, \\
a_{5}=0.3151, & b_{5}=0.3468, \\
a_{6}=0.6334, & b_{6}=0.6654, \\
a_{7}=0.9565, & b_{7}=0.9892 .
\end{aligned}
$$

Observe that for $i=1,2,3,4,5, b_{i+2}(8)-a_{i}(8) \leq b_{7}(8)-a_{5}(8)=0.8968<1$. Also, for $i=1,2,3, a_{i+4}(6)-b_{i}(6) \geq a_{5}(6)-b_{1}(6)=1.2274>1$. Consider the axis-aligned square $U_{4}$ of side 2 centered at $\xi_{4}=\left(\left(q_{l}(6)+q_{r}(6)\right) / 2,7\right)$. We have

$$
q_{r}(6)-q_{l}(6) \geq a_{5}(6)-b_{1}(6)=1.2274>1 \text {. }
$$

It follows in the same way that $U_{4}$ is empty of disk centers, in contradiction with Lemma 2.

Case 3: There are no three consecutive monochromatic points on $\ell$, and the red span is at 5 or 6 . Let $l<r$ be the two corresponding indices, and observe again (as in the previous case) that the ray through $q_{l}$ has negative slope, and the ray through $q_{r}$ has negative slope. Consider the two lines $\ell(6)$ and $\ell(4)$. The corresponding values for $a_{i}(y)$ and $b_{i}(y), y=4$, are:

$$
\begin{aligned}
y=4: \quad a_{1} & =-0.6273, \quad b_{1}=-0.6063, \\
a_{2} & =-0.4177, \quad b_{2}=-0.3968, \\
a_{3} & =-0.2089, \quad b_{3}=-0.1881, \\
a_{4} & =0.0000, \quad b_{4}=0.0209, \\
a_{5} & =0.2103, \quad b_{5}=0.2315,
\end{aligned}
$$




$$
\begin{array}{ll}
a_{6}=0.4232, & b_{6}=0.4446, \\
a_{7}=0.6398, & b_{7}=0.6617 .
\end{array}
$$

Observe that for $i=1,2,3,4, b_{i+3}(6)-a_{i}(6) \leq b_{7}(6)-a_{4}(6)=0.9892<1$. Also, for $i=1,2, a_{i+5}(4)-b_{i}(4) \geq a_{6}(4)-b_{1}(4)=1.0294>1$. Consider the axisaligned square $U_{5}$ of side 2 centered at $\xi_{5}=\left(\left(q_{l}(4)+q_{r}(4)\right) / 2,5\right)$. We have

$$
q_{r}(4)-q_{l}(4) \geq a_{6}(4)-b_{1}(4)=1.0294>1 .
$$

It follows as before that $U_{5}$ is empty of disk centers, in contradiction with Lemma 2.

This completes the case analysis and thereby the proof of Lemma 8.

\subsection{Putting It All Together: Proof of Theorem 1}

By Lemma 3 there are at least $3 L^{2} / 16$ disks from $F$ in $\Omega_{1}$. By Lemma 8 , no disk in $\Omega_{1}$ is tangentially illuminated, so by Lemma 7 the total number of disks in $\Omega_{1}$ that are totally illuminated is at most $3 L^{2} / 20$. It follows that at least

$$
\frac{3 L^{2}}{16}-\frac{3 L^{2}}{20}=\frac{3 L^{2}}{80} \geq 1.96 \cdot \frac{3 R^{2}}{80} \geq \frac{R^{2}}{14}
$$

disks in $\Omega_{1}$, thus also in $\Omega$, are only partially illuminated, and therefore each has a hidden point on its boundary. We conclude that for large enough $R$, there exist many distinct hidden points, each associated with a different disk in $F$. It is enough to choose $R$ so that the condition in Lemma 7 is satisfied, namely

$$
R \geq \frac{760 \cdot 5^{151}}{0.99 \sqrt{2}}=1.9018 \ldots \times 10^{108} .
$$

Taking $R \geq 2 \cdot 10^{108}$ will do, and the proof of Theorem 1 is complete.

Discussion on parameters selection Most of the parameters are determined by $\phi=\pi / 600$ and $\beta=\phi / 2=\pi / 1200$. The choice of the "wiggle angle" $\phi=\pi / 600$ is needed in Lemma 8, and the value of the "classification angle" $\beta$ chosen here is convenient for Lemma 1 . The value $\theta=\pi / 60$ is one that works in Lemma 8 .

\section{Proof of Theorem 2}

Let $F=\left\{\omega_{1}, \ldots, \omega_{n}\right\}$ be the set of $n$ disks contained in $\Omega$. Each pair of disks in $F$ admits four common tangent segments; see Fig. 1. Let $\mathcal{T}$ denote the set of all $O\left(n^{2}\right)$ tangent segments. If a tangent segment intersects any of the other $n-2$ disks, it is subsequently discarded (such as the rightmost tangent in Fig. 1). Let $\mathcal{T}^{\prime}$ denote the resulting set; clearly $\left|\mathcal{T}^{\prime}\right|=O\left(n^{2}\right)$. For $s \in \mathcal{T}^{\prime}$, let $\ell(s)$ denote the supporting line of $s$. Each tangent segment in $\mathcal{T}^{\prime}$ is extended both ways until it hits one of the disks or to infinity (equivalently, until it hits the enclosing disk $\Omega$ ). Since $\left|\mathcal{T}^{\prime}\right|=O\left(n^{2}\right)$, we obtain a collection $\mathcal{I}$ of $O\left(n^{2}\right)$ circular elementary intervals on the boundaries of the 
disks, that is, intervals without any other tangent with disk intersection points in their interior. The sets $\mathcal{T}^{\prime}$ and $\mathcal{I}$ can be computed in $O\left(n^{5 / 2} \log n\right)$ time as follows. We use the ray shooting data structure of Agarwal et al. [1, Theorem 7.1], building on an earlier method of van Kreveld et al. [8].

The data structure $\mathcal{D}$ stores a collection of $n$ pairwise disjoint disks, for answering ray shooting queries: for a query ray $\rho$, the first disk hit by the ray is returned as the answer to the query. $\mathcal{D}$ can be constructed in $O\left(n^{2} \log n\right)$ time and uses $O(n \log n)$ space. The query processing time is $O(\sqrt{n} \log n)$. For each element $s \in \mathcal{T}$, three queries with rays collinear with $\ell(s)$ are performed: the rays are anchored at the two endpoints of $s$. The first query determines if $s$ is discarded, and if the answer is negative, i.e., $s \in \mathcal{T}^{\prime}$, the remaining two queries find the intersection points with the first disk hit or with $\partial \Omega$, in either direction. Since there are $O\left(n^{2}\right)$ queries overall, they can be processed in $O\left(n^{5 / 2} \log n\right)$ time. Once all queries have been answered, for each disk $\omega \in F$, the circular order of the intersection points on its boundary is computed to find the elementary intervals. This takes overall $O\left(n^{2} \log n\right)$ time (for all $n$ disks). Observe that all points in an elementary interval are equivalent with respect to illumination. Note also that each of the $O\left(n^{2}\right)$ endpoints of the elementary intervals determines a dark/illuminated labeling for the left and right adjacent intervals, as a function of the position of the tangent line relative to the two disks involved. This information can be used to determine the status (dark versus illuminated) of each interval in $\mathcal{I}$ with no additional time overhead. The resulting time complexity of the algorithm is therefore dominated by the overall query processing time, hence it is $O\left(n^{5 / 2} \log n\right)$.

Remark One can construct small examples of a dense forest with congruent (unit radius) disks with interior hidden points but no boundary hidden points. See Fig. 10: the construction is symmetric with respect to the two coordinate axes, with the center of the rightmost disk at $\left(\frac{16}{7}, 0\right)$, and the center of the top disk at $\left(0, \frac{17}{16}\right)$. Let $\alpha$ be the angle of the tangent line (of positive slope) through the origin $o=(0,0)$ to the rightmost disk, as shown in the figure. Let $\beta$ be the angle of the tangent line (of positive slope) through the origin $o=(0,0)$ to the top disk, as shown in the figure. By construction, we have $\sin \alpha=\frac{7}{16}$ and $\cos \beta=\frac{16}{17}$. To see that the origin $o$ is dark, it is enough to check that $\beta<\alpha$ or, equivalently, that $\sin \beta<\sin \alpha: \frac{\sqrt{33}}{17}<\frac{7}{16}$. Similarly, one can verify that all boundary points are illuminated, by computing the angles made by the two tangent lines through the point $\left(0, \frac{1}{16}\right)$ to the rightmost and to the lowest disk.

By Theorem 1, there are no such examples if the forest radius is large enough (i.e., $R \geq 2 \cdot 10^{108}$ ). However if one gives up the density constraint, the example in Fig. 10 can be extended for an arbitrary large number of congruent disks. This also brings up the question whether computing the (entire) illumination map of a forest is harder than computing the boundary illumination map. Note that computing the arrangement of tangent lines with the same idea as in the proof of Theorem 2 gives a polynomial-time algorithm; however its time complexity is higher. 
Fig. 10 An interior hidden point (filled circle) in a dense forest with four congruent trees and no boundary hidden point

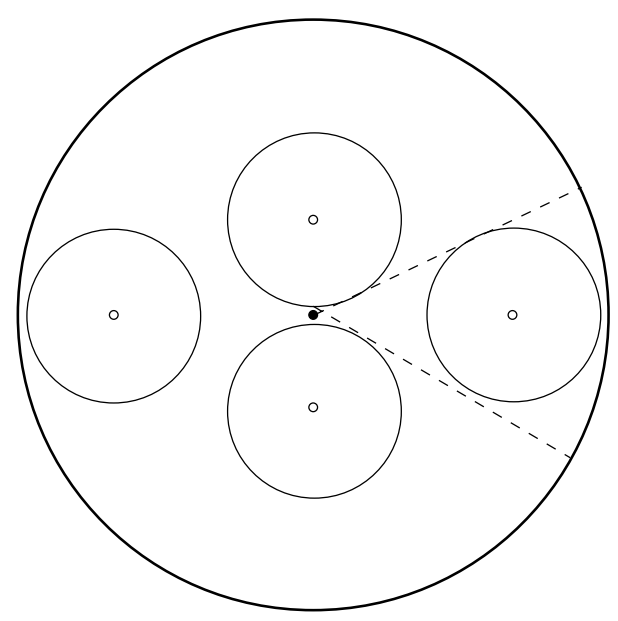

\section{Appendix A: Source Code}

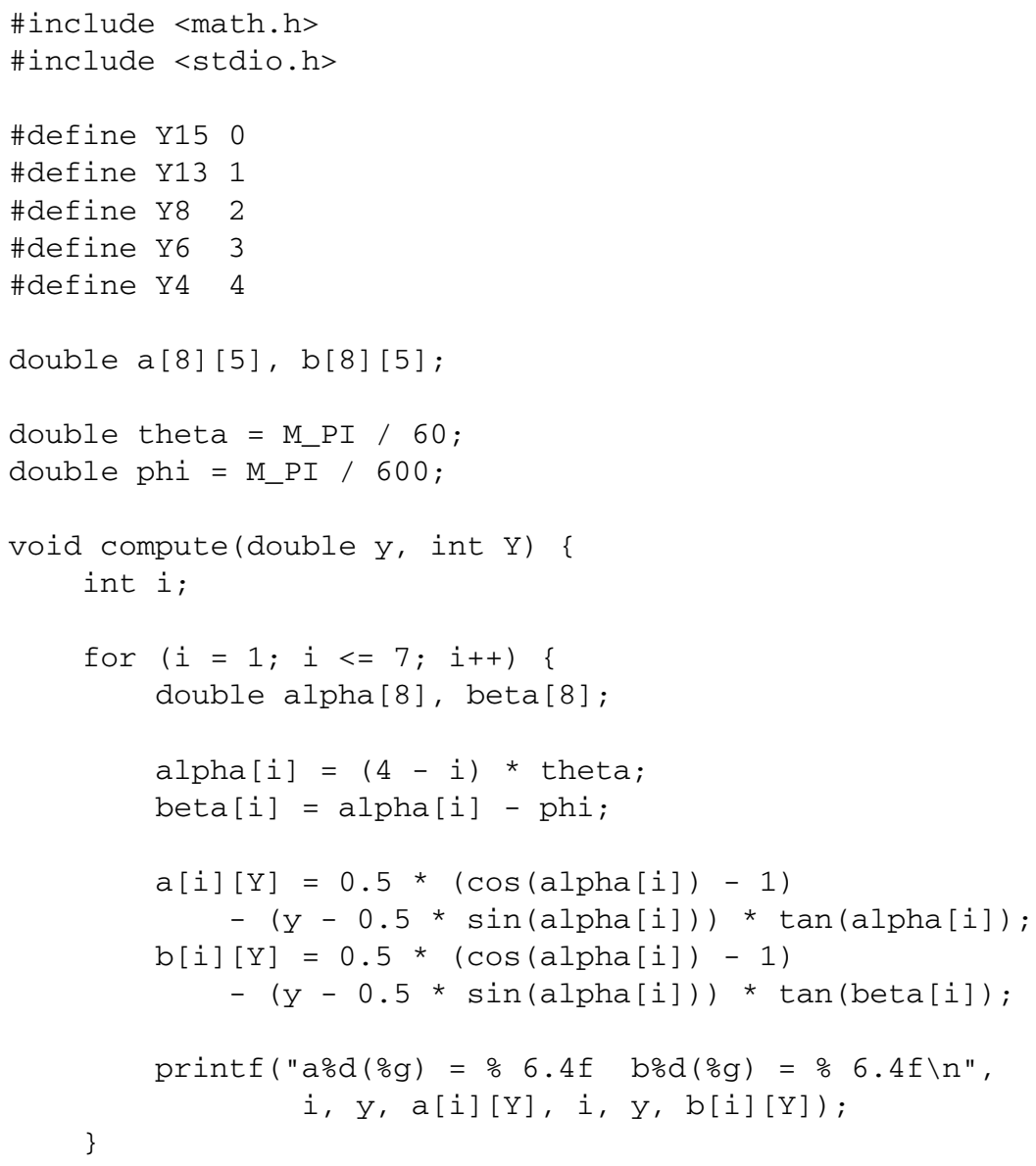




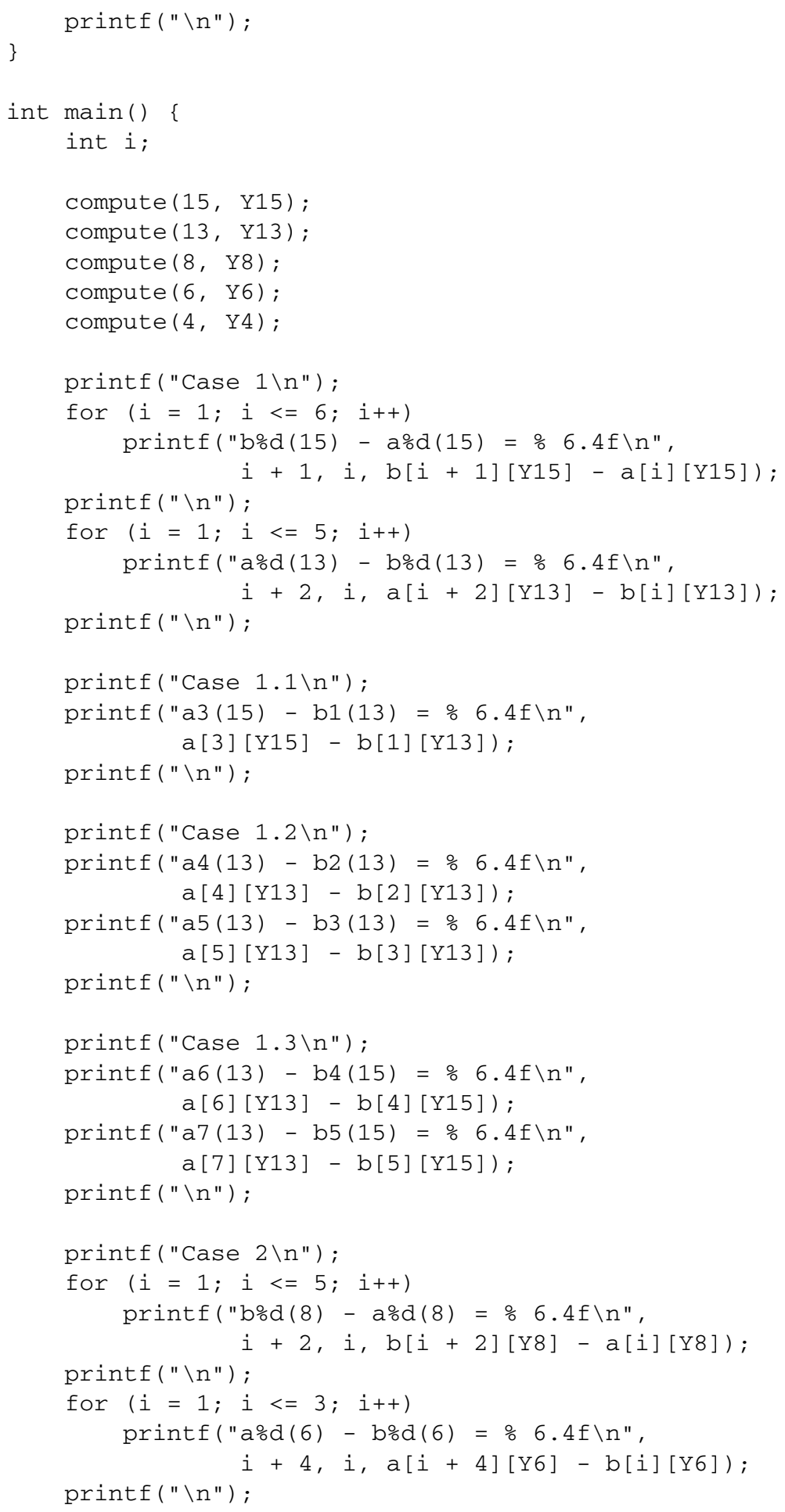




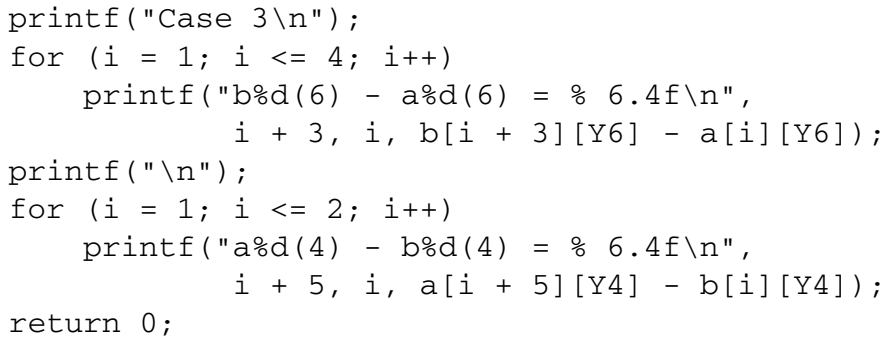

\section{Appendix B: Output}

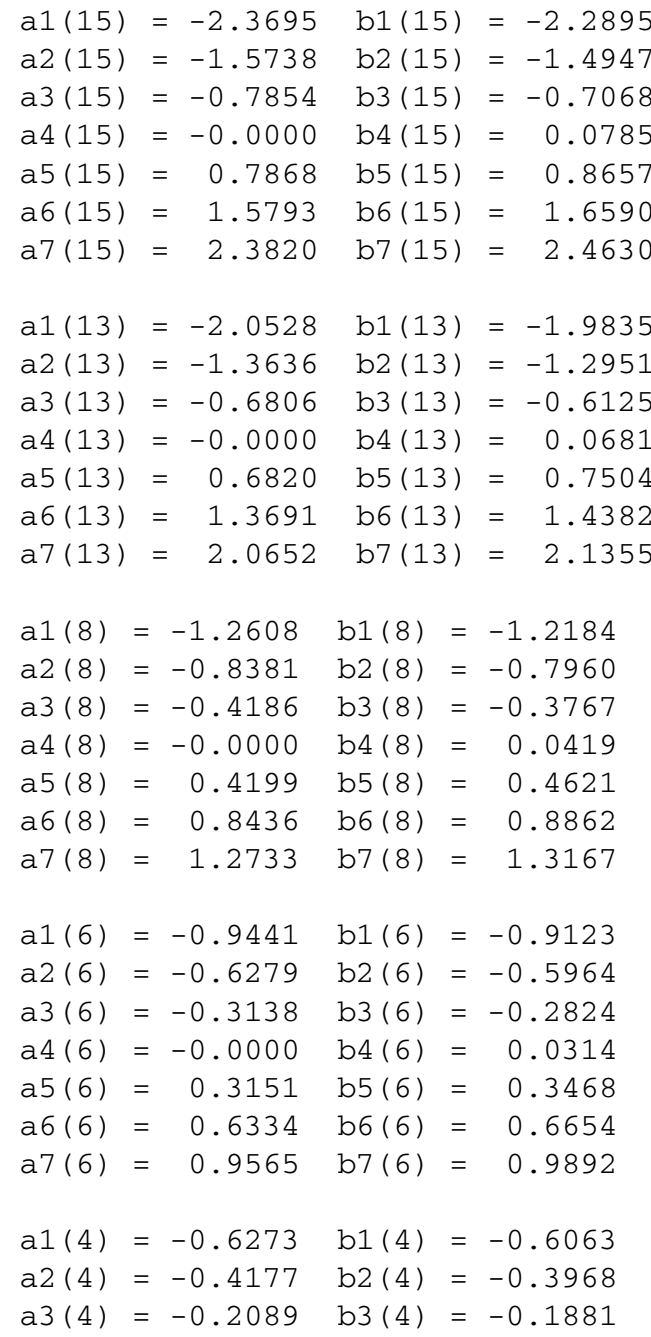




$\mathrm{a} 4(4)=-0.0000 \mathrm{~b} 4(4)=0.0209$
$\mathrm{a} 5(4)=0.2103 \mathrm{~b} 5(4)=0.2315$
$\mathrm{a} 6(4)=0.4232 \mathrm{~b} 6(4)=0.4446$
$\mathrm{a} 7(4)=0.6398 \mathrm{~b} 7(4)=0.6617$

Case 1
$\mathrm{~b} 2(15)-\mathrm{a} 1(15)=0.8748$
$\mathrm{~b} 3(15)-\mathrm{a} 2(15)=0.8670$
$\mathrm{~b} 4(15)-\mathrm{a} 3(15)=0.8640$
$\mathrm{~b} 5(15)-\mathrm{a} 4(15)=0.8657$
$\mathrm{~b} 6(15)-\mathrm{a} 5(15)=0.8722$
$\mathrm{~b} 7(15)-\mathrm{a} 6(15)=0.8837$
$\mathrm{a} 3(13)-\mathrm{b} 1(13)=1.3029$
$\mathrm{a} 4(13)-\mathrm{b} 2(13)=1.2951$
$\mathrm{a} 5(13)-\mathrm{b} 3(13)=1.2945$
$\mathrm{a} 6(13)-\mathrm{b} 4(13)=1.3010$
$\mathrm{a} 7(13)-\mathrm{b} 5(13)=1.3148$

Case 1.1

$\mathrm{a} 3(15)-\mathrm{b} 1(13)=1.1980$

Case 1.2

$\mathrm{a} 4(13)-\mathrm{b} 2(13)=1.2951$

$\mathrm{a} 5(13)-\mathrm{b} 3(13)=1.2945$

Case 1.3

$\mathrm{a} 6(13)-\mathrm{b} 4(15)=1.2906$

$\mathrm{a} 7(13)-\mathrm{b} 5(15)=1.1995$

Case 2

$\mathrm{b} 3(8)-\mathrm{a} 1(8)=0.8841$

$\mathrm{b} 4(8)-\mathrm{a} 2(8)=0.8800$

$\mathrm{b} 5(8)-\mathrm{a} 3(8)=0.8807$

$\mathrm{b} 6(8)-\mathrm{a} 4(8)=0.8862$

$\mathrm{b} 7(8)-\mathrm{a} 5(8)=0.8968$

$\mathrm{a} 5(6)-\mathrm{b} 1(6)=1.2274$

$\mathrm{a} 6(6)-\mathrm{b} 2(6)=1.2298$

$\mathrm{a} 7(6)-\mathrm{b} 3(6)=1.2389$

Case 3

$\mathrm{b} 4(6)-\mathrm{a} 1(6)=0.9755$

$\mathrm{b} 5(6)-\mathrm{a} 2(6)=0.9747$

$\mathrm{b} 6(6)-\mathrm{a} 3(6)=0.9792$

$\mathrm{b} 7(6)-\mathrm{a} 4(6)=0.9892$

$\mathrm{a} 6(4)-\mathrm{b} 1(4)=1.0294$

$\mathrm{a} 7(4)-\mathrm{b} 2(4)=1.0365$ 


\section{References}

1. Agarwal, P.K., van Kreveld, M., Overmars, M.: Intersection queries in curved objects. J. Algorithms 15(2), 229-266 (1993)

2. Allen, T.T.: Pólya's orchard problem. Am. Math. Mon. 93(2), 98-104 (1986)

3. Croft, H.T., Falconer, K.J., Guy, R.K.: Unsolved Problems in Geometry. Springer, New York (1991)

4. Hardy, G.: Ramanujan: Twelve Lectures on Subjects Suggested by His Life and Work, 3rd edn. Chelsea, New York (1999)

5. Honsberger, R.: The orchard problem. In: Mathematical Gems. Math. Assoc. America, Washington (1973)

6. Jovanović, N., Korst, J., Clout, R., Pronk, V., Tolhuizen, L.: Candle in the woods: asymptotic bounds on minimum blocking sets. In: Proceedings of the 25th Annual Symposium on Computational Geometry, pp. 148-152 (2009)

7. Jovanović, N., Korst, J., Janssen, A.J.: Minimum blocking sets of circles for a set of lines in the plane. In: Proceedings of the 20th Canadian Conference on Computational Geometry, pp. 91-94 (2008)

8. van Kreveld, M., Overmars, M., Agarwal, P.K.: Intersection queries in sets of disks. BIT 32(2), 268279 (1992)

9. Kruskal, C.: The orchard visibility problem and some variants. J. Comput. Syst. Sci. 74, 587-597 (2008)

10. Mitchell, J.: Dark points among disks. In: Open Problems from the 2007 Fall Workshop in Computational Geometry. http://www.research.ibm.com/people/l/lenchner/fwcg2007/ fwcg_open_problems.pdf

11. O'Rourke, J.: Visibility. In: Goodman, J., O'Rourke, J. (eds.) Handbook of Discrete and Computational Geometry, 2nd edn., pp. 643-663. Chapman \& Hall, London (2004)

12. O'Rourke, J., Petrovici, O.: Narrowing light rays with mirrors. In: Proceedings of the 13th Canadian Conference on Computational Geometry, pp. 137-140 (2001)

13. Polya, G.: Zahlentheoretisches und wahrscheinlichkeitstheoretisches über die Sichweite im Walde. Arch. Math. Phys. Ser. 2 27, 135-142 (1918)

14. Polya, G., Szego, G.: Problems and Theorems in Analysis, vol. II. Springer, New York (1976). Chapter 5, Problem 239

15. Yaglom, A.M., Yaglom, I.M.: Challenging Mathematical Problems with Elementary Solutions, vol. II. Holden-Day, Oakland (1967). Translated by James Mc-Cawley, Jr., revised and edited by Basil Gordan 